\title{
Performance Evolution for Frequency Invariance for Double Differential Detection System
}

\author{
${ }^{1}$ SudhaRani karedla, ${ }^{2}$ Sri. E.V. Narayana \\ ${ }^{1,2}$ Electronics and Communications Engineering, JNTU, Kakinada, India
}

\begin{abstract}
The Evolution of Multiple symbol differential detection (MSDD) of double differential QAM signal is studied in the presence of frequency offset, phase offset and additive white Gaussian noise. It is shown that in the case of nonzero frequency offset distorts the transmitted signal through attenuating its amplitude and MSDD receiver degrades by increases the number of samples. MSDD signal makes use of maximum likehood sequence estimation of transmitted phase rather than the symbol by symbol detection as in the conventional differential detection. It is shown that the proposed receiver is robust to the distortions caused by the random frequency variations and a lower bound on the error probability of the proposed MSDD receiver is also derived .
\end{abstract}

Keywords-Differential encoding, maximum-likelihood detection, multiple symbol differential detection, Quadrature Amplitude Modulation.

\section{Introduction}

It is well known that, in applications where simplicity and robust of implementation take precedence over achieving the best system performance, differential detection is an attractive alternative to coherent detection .Aside from implementation considerations. It is also possible that the transmission environment may be sufficiently degraded, e.g.., a multipath fading channel , that acquiring and tracking a coherent demodulation reference signal is difficult if not impossible.

In the past, differential detection or multiple Quadrature Amplitude Modulation (MQAM) has been accomplished by comparing the received phase in a given symbol interval to that in the previous symbol interval and making a multilevel decision on the difference between these two phases. Differential detection of Quadrature Amplitude Modulation (QAM) signals is a well-known strategy for mitigating the performance degradation due to unknown phase offset. The constellation rotation caused by the phase offset can be removed using a differential QAM (DQAM) modulation scheme along with a differential detector[1]. However, this detector suffers from a signal-to-noise power ratio (SNR) loss compared to a coherent detector[1]. An effective means to mitigate this SNR loss is known as multiple-symbol differential detection (MSDD) [2]-[4].

The MSDD scheme is, indeed, a more general case of the conventional differential detection in which more than two consecutive samples are utilized to detect the information symbols. It is shown in [2] that by increasing the number of received samples in MSDD, the receiver performance approaches that of coherent demodulation of DQAM signals.

In this paper, we study the effect of frequency variations on a QAM signal transmitted over an additive white Gaussian noise (AWGN) channel. We show that frequency offset attenuates the amplitude of the transmitted signal and rotates its constellation points about the origin by a time-varying phase. Then, we derive a MSDD scheme to demodulate a DDQAM signal and show that this scheme is not sensitive to constellation rotation caused by the frequency offset.

The proposed demodulator suffers from a SNR loss compared to a conventional MSDD with DQAM modulation when frequency offset is not present. However, as will be seen in the sequel, the SNR loss can be less than $3 \mathrm{~dB}$ for some modulation schemes resulting in a net performance gain relative to the ACD-based MSDD scheme proposed [10].

This paper is organized as follows. In Section II, we present system model and maximum-likehood detection of MQAM. In Section III, we propose a new MSDD scheme for DDQAM signal and derive a lower bound on its bit error probability. Numerical results are presented in Section IV. In Section V, some conclusion are drawn.

\section{System Model And Maximum-Likehood Detection Of MQAM}

Consider communication over an additive white Gaussian noise (AWGN) channel. Assuming perfect symbol timing at the receiver, the baseband equivalent received signal can be expressed as

$$
r_{k}=S_{k} e^{j(k \Psi+\emptyset)}+n_{k} \quad(k-1) \mathrm{T} \leq \mathrm{t} \leq \mathrm{kT}
$$

Where $\Psi=2 \Pi f_{0} T$,Skis theinformation symbol at the $k^{t h}$ symbol interval, $n_{k}$ is the AWGN sample at the $k^{\text {th }}$ symbol interval with zero mean and variance 


$$
\boldsymbol{\sigma}_{\mathbf{n}}^{2}=\frac{1}{2} \mathbf{E}\left\{\mathbf{n}_{\mathbf{k}} \mathbf{n}_{\mathbf{k}}^{*}\right\}=\frac{\mathbf{N}_{\mathbf{0}}}{\mathbf{T}}
$$

$\mathrm{T}$ is the symbol interval and $f_{o}$ and $\phi$ are the frequency offset and phase offset, respectively,. Assumenow ,a well known DQAM modulation scheme is used to modulate the information symbols, then the $k^{\text {th }}$ transmitted symbol can be written as

$$
S_{k}=\sqrt{2 P} e^{\theta_{k}}
$$

Where $\mathrm{P}$ is the transmitter's power and $\theta_{k} \in\{2 \pi m / M\}_{m=0}^{M-1}$ is the transmitted phase at the $k^{\text {th }}$ symbol interval and $M$ denotes size of the constellation.

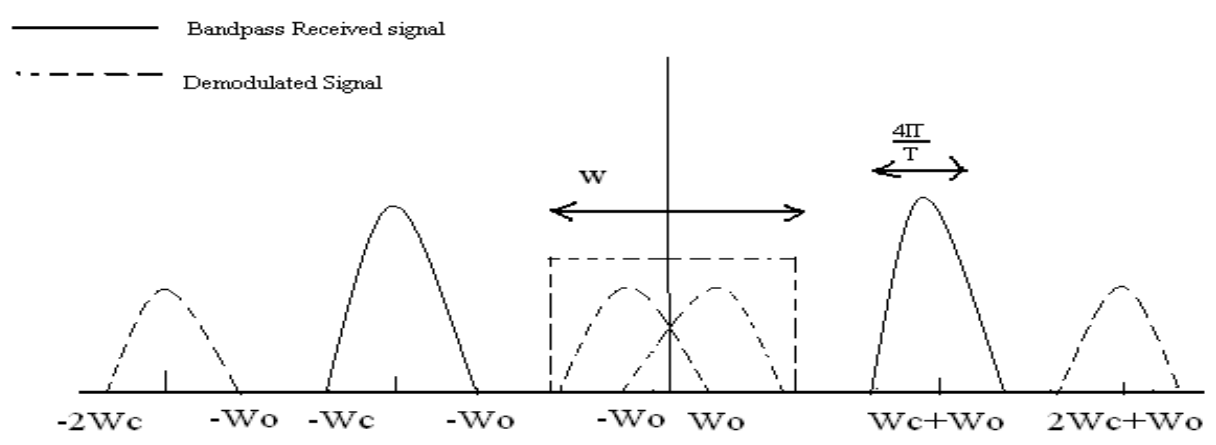

Fig. 1. The frequency domain representation of a band limited received signal, and its demodulated version in the presence of frequency offset.

In contrast, the constellation rotation caused by the frequency offset can degrade the performance rather rapidly even for small values of $\psi$. This is because the angle of rotation is a function of time and varies from one symbol interval to the other. The time-varying constellation rotation can be converted to a time invariant rotation by using a DQAM modulation scheme. In a DQAM modulation scheme the $k^{\text {th }}$ transmitted phase is defined as

$$
\boldsymbol{\theta}_{\boldsymbol{k}} \triangleq \sum_{i=\mathbf{1}}^{\boldsymbol{k}} \dot{\boldsymbol{\theta}}_{\boldsymbol{i}}
$$

Where $\dot{\theta}_{i}$ is the $i$ th uncoded information phase and clearly $\dot{\theta}_{i}$ can be written as $\dot{\theta}_{i}=\theta i-\theta i-1$. Assume that the term $\exp (j k \psi)$ in (1) is absorbed into $S_{K}$. Then, eq(1) can be written as

$$
r_{k}=v_{k} e^{j \emptyset}+n_{k}
$$

Where

$$
\begin{aligned}
& v_{k} \triangleq \sqrt{2 P}+e^{j\left(\theta_{k}+k \Psi\right)} \\
& v_{k}=\sqrt{2 P} \exp \left[j \sum_{i=1}^{k}\left(\dot{\theta}_{\mathrm{i}}+\Psi\right)\right]
\end{aligned}
$$

This means that in the presence of the frequency offset, the information phases should be chosen from $\{2 \pi m / M+\psi\} M-1 m=0$ rather than $\{2 \pi m / M\} M-1 m=0$. In other words, the effect of $\psi$ on a DQAM signal is similar to the effect of phase offset on a QAM signal. Therefore, a DDQAM modulation should be invariant to frequency offset as the DQAM modulation is invariant to phase offset.

\section{Frequency Offset Insensitive Differential Detection}

A . Receiver Derivation:

Assume that the frequency offset and the phase offset are constant over $N$ successive samples. Then, using (4) one can define a new variable $r$ as

$$
\breve{\mathbf{r}}_{\mathbf{k}} \triangleq \mathbf{r}_{\mathbf{k}} \mathbf{r}_{\mathbf{k}-1}^{*}
$$




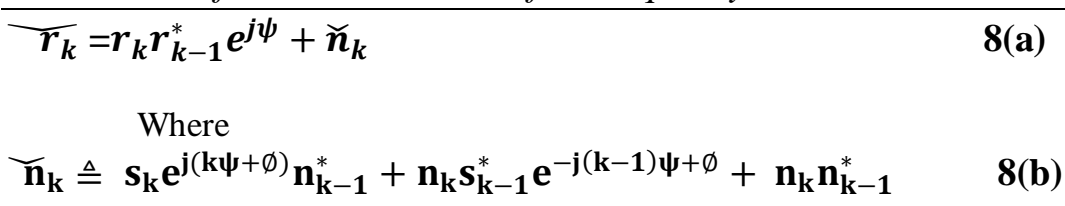

Assume that a QAM modulation is employed, i.e., $r_{k}$ is given by (1). Then, the first and second terms on the right of (8b) are independent Gaussian random variables each with mean zero and variance $\sigma_{n}^{2}$. In contrast, the last term has a complicated probability density function[12]. However, for practical SNR values this term is relatively small compared to the first two and can be ignored. Moreover, it can be shown that $\breve{n}_{k}$ and $\breve{n}_{l}$ are uncorrelated for $k \neq \ell$. Therefore, we approximate $\left\{\breve{n}_{k}\right\}$ as a zero-mean Gaussian random sequence with

$$
E\left\{\dot{n}_{k} \dot{n}_{l}^{*}\right\}=\sigma_{n}^{2} \delta(k-\ell)
$$

Where $(\cdot)$ denotes the Kronecker delta function.

Assume now that $\psi$ has a uniform distribution over the interval $[0,2 \pi)$ and that any amplitude variations due to frequency offset can be ignored. Also assume that in (8a) the

term $P_{k} P_{k-1}^{*}=\varepsilon_{S} \exp \left(\mathrm{j} \dot{\theta}_{k}\right)$ denotes the $k_{t h}$ transmittedsymbol. Then, the maximum-likelihood (ML) receiver should maximize the metric[2].

$$
\begin{aligned}
\Lambda & =\left|\sum_{\mathbf{i}=\mathbf{0}}^{\mathrm{N}-1} \check{\mathbf{r}}_{\mathrm{k}-\mathrm{i}} \mathbf{e}^{-\mathrm{j} \dot{\theta}_{\mathrm{k}-\mathrm{i}}}\right|^{2} \\
& =\left|\sum_{\mathbf{i}=\mathbf{0}}^{\mathrm{N}-1} \check{\mathbf{r}}_{\mathrm{k}-\mathrm{i}} \mathbf{e}^{-\mathbf{j}\left(\dot{\theta}_{\mathrm{k}-\mathrm{i}}-\dot{\theta}_{\mathrm{k}}-\mathrm{N}+1\right.}\right|^{2}
\end{aligned}
$$

Where the last equation follows from the fact that multiplying the argument of the |. |in (10) by $\dot{\theta}_{k-N+1}$ does not change the metric[2]. Note that in this case we need $\mathrm{N}+1$ samples to detect $\mathrm{N}-1$ information symbols. It can be readily verified that

$$
\boldsymbol{\theta}_{k-i}-\dot{\boldsymbol{\theta}}_{\boldsymbol{k}-N+1}=\sum_{m=0}^{N-i-2} \ddot{\theta}_{k-i-m}
$$

Where now $\ddot{\theta}_{k}=\dot{\theta}_{k}-\dot{\theta}_{k-1}$ is the information phase at the $k$ th time interval. Thus, (11) can be rewritten as

$$
=\left|\check{\mathbf{r}}_{\mathrm{k}-\mathrm{N}-1}+\sum_{\mathrm{i}=\mathbf{0}}^{\mathrm{N}-2} \check{\mathbf{r}}_{\mathrm{k}-\mathrm{i}} \exp \left[-\mathbf{j} \sum_{\mathrm{m}=\mathbf{0}}^{\mathrm{N}-\mathbf{i}-2} \ddot{\theta}_{\mathrm{k}-\mathbf{i}-\mathrm{m}}\right]\right|^{2}
$$

Clearly, the metric in (13) is independent of the frequency offset and the phase offset. Assuming that $\theta_{-1}=\theta_{o}=$ 0 and

$$
\begin{aligned}
\boldsymbol{\theta}_{k} & =\sum_{i=1}^{k}(k-i+1) \ddot{\theta}_{i} \\
& =\underbrace{\sum_{i-1}^{k} \overbrace{\sum_{m=1}^{i} \ddot{\theta}_{m}}}
\end{aligned}
$$

Encoding the information symbols using (14) is equivalent to encoding the information symbols using a DQAM encoder once, and then encoding the resulting symbols one more time with the same encoding rule. Note that the analysis presented in this section serves as the proof of optimality for the MSDD receiver when the $\left\{\check{n}_{k}\right\}$ are Gaussian. This occurs when the cross-noise term in (8b) is negligible, i.e., when the SNR is relatively large.

\section{B. Bit Error Probability performance:}

For DDQAM signals, the I-Q demodulator presented in[10,Section V] is a special case of the proposed receiver when $N=2$. In the absence of the frequency offset, an exact expression for the BER of the IQ demodulator with binary DDQAM modulation has been derived in [8],[13]. For higher order DDQAM signals, i.e., when $M>2$, an exact expression for the BER of the MSDD is not known to the best of authors' knowledge. However, an upper bound for the symbol error rate (SER) for the case where $N=2$ has been derived in $[8],[13]$. 
We now obtain a reasonably tight lower bound on the BER of the MSDD for the case where $N \rightarrow \infty$. To this end, we recall a fact presented in [2] that the performance of the MSDD with DQAM modulation and $N \rightarrow \infty$, is lower bounded by that of the coherent detection of differentially encoded QAM [14, eq. (8.36)],2 i.e.,

$$
\mathbf{P}_{\mathrm{S}}^{\mathrm{LB}}=\mathbf{P}_{\mathrm{s}}\left(2-\mathbf{P}_{\mathrm{s}}\right)-\sum_{\mathrm{m}=1}^{\mathrm{M}-1} \mathbf{P}_{\mathrm{m}}^{2}
$$

where $P_{m}$ is the probability that the AWGN moves the transmitted $M$ QAM symbol $m$ decision regions away from the correct region and $P \mathrm{~s}$ is the SER of the optimum decision rule for detecting a single symbol transmitted over an AWGN channel for QAM,DQAM and DDQAM modulation is given by

$$
\begin{aligned}
& \text { QAM: } \quad \arg _{\theta_{k}}^{\max }\left\{\boldsymbol{r}_{\boldsymbol{k}} \boldsymbol{e}^{-j \theta_{k}}\right\} \\
& \text { DQAM: } \quad \arg _{\theta_{k}}^{\max }\left\{r_{k} r_{k-1}^{*} e^{-j \theta_{k}}\right\} \\
& \text { DDQAM: } \arg _{\theta_{k}}^{\max }\left\{r_{k} r_{k-1}^{*}\left(r_{k-1} r_{k-2}^{*}\right) e^{-j \theta_{k}}\right\}
\end{aligned}
$$

The lower bound in (18) is valid only when the received samples, $\left\{r_{k}\right\}$, are used to evaluate the MSDD's decision metric. However, the receiver uses the $\left\{\tilde{r}_{k}\right\}$ to evaluate the decision metric given by (11). Therefore, the lower bound in (15) should be adjusted to account for the above fact. To this end, by replacing $r_{k}$ with $\tilde{r}_{k}$ in (18), one has that

$$
\arg _{\theta_{\mathrm{k}}}^{\max }\left\{\tilde{\mathbf{r}}_{\mathbf{k}} \mathbf{e}^{-\mathbf{j} \theta_{\mathrm{k}}}\right\}
$$

Eq. (19) denotes the decision rule of the optimum differential detector of MDQAM when $N=2$. The SER of this receiver is given by [15 eq. (3)]

$$
P_{S}=\frac{1}{\pi} \int_{0}^{\pi-\pi / M} \exp \left(\frac{-\gamma_{S} \sin ^{2}\left(\frac{\pi}{M}\right)}{1+\cos \left(\frac{\pi}{M}\right) \cos \theta}\right) d \theta(20)
$$

We also use the distribution of the phase angle between two vectors corrupted by uncorrelated Gaussian noise given to obtain $P m$, after some manipulations, as

$$
\begin{gathered}
P_{m}=\frac{1}{2 \pi} \int_{0}^{\pi-\frac{(2 m-1) \pi}{M}} \exp \left(\frac{-\gamma_{s} \sin ^{2}\left(\frac{(2 m-1) \pi}{M}\right)}{1+\cos \left(\frac{(2 m+1) \pi}{M}\right) \cos \theta}\right) d \theta \\
-\frac{1}{2 \pi} \int_{0}^{\pi-\frac{(2 m+1) \pi}{M}} \exp \left(\frac{-\gamma_{s} \sin ^{2}\left(\frac{(2 m+1) \pi}{M}\right)}{1+\cos \left(\frac{(2 m+1) \pi}{M}\right) \cos \theta}\right) d \theta \\
m=1,2, \ldots \ldots . . . . \mathrm{M}-1 .
\end{gathered}
$$

For the special case when $M=2, P_{b}^{L B}$ can be expressed in closed-form as

$$
\mathbf{P}_{\mathbf{b}}^{\mathrm{LB}}=\mathbf{e}^{-\gamma \mathbf{b}}-\frac{1}{2} \mathbf{e}^{-2 \gamma_{b}}
$$

Where $\gamma b$ denotes the SNR per bit. Note that for moderate to large values of SNR the square terms on the right of (19) are very small and can be ignored. Hence, for these SNR values $P$ LB s is approximately equal to $2 P s$.

\section{Numerical Results}

As mentioned in Section II, the expression given in (1) is an approximation for the sampled received signal as it does not account for the amplitude attenuation caused by the frequency offset. It is, therefore, important to examine the receiver performance in the presence of this attenuation and indicate how good the aforementioned approximation is. To this end, we evaluate the error probability performance of the proposed MSDD receiver as a function of $\psi$ for $\gamma b=10 \mathrm{~dB}$ and selected values of $N$. We assume a rectangular pulseshaping filter is used at the transmitter. The results are shown in Fig.2 for binary DDQAM (BDDQAM) and 


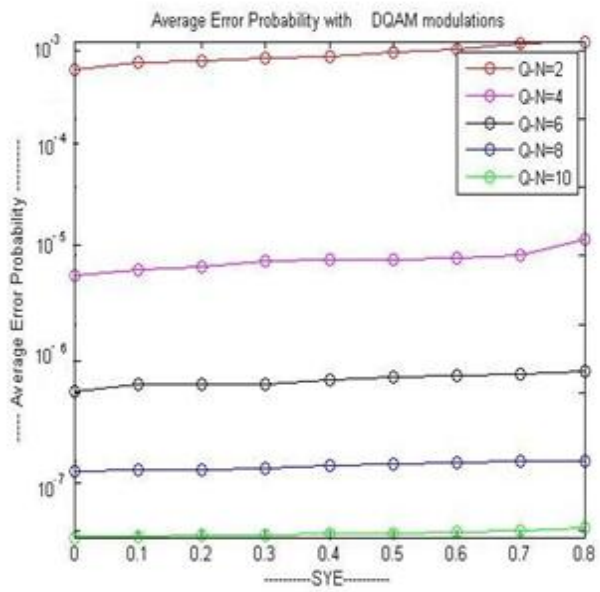

Fig.2.The average error probability of the proposed MSDD as a function of DQAM modulation for $\mathrm{SNR}=10 \mathrm{~dB}$ and $\mathrm{N}=2,4,6,8$ and 10.

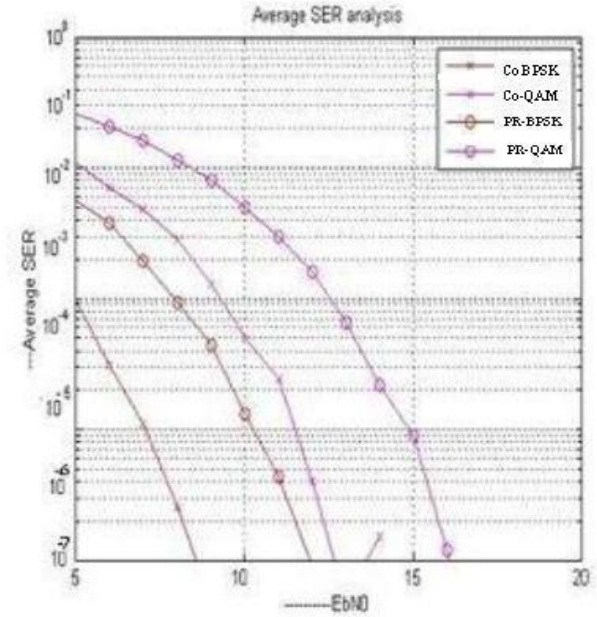

Fig. 3. The lower bound on the SER of the proposed receiver and the average SER for coherent detection of BQAM and QAM as a function of SNR for $M=2,4,8$.

Fig. 3 illustrates the error probability performance of the proposed receiver DQAM with QDDPSK modulation for the cases where $\psi=0$, and selected values of $N$. As seen in the figure, the performance of the receiver is approximately the same for both cases. Note that in this case, increasing the observation interval significantly improves the receiver performance. For example, when the SER $=10-5$, increasing $N$ from 2 to 10 leads to a SNR gain of $3.5 \mathrm{~dB}$. It is shown in [10] that the ACD receiver suffers from a $3 \mathrm{~dB}$ SNR loss compared to the coherent detection of DQAM signals when $N \rightarrow \infty$ and SNR is very large. It is, therefore, desirable to investigate the SNR loss of the proposed receiver relative to the coherent detection of DQAM.

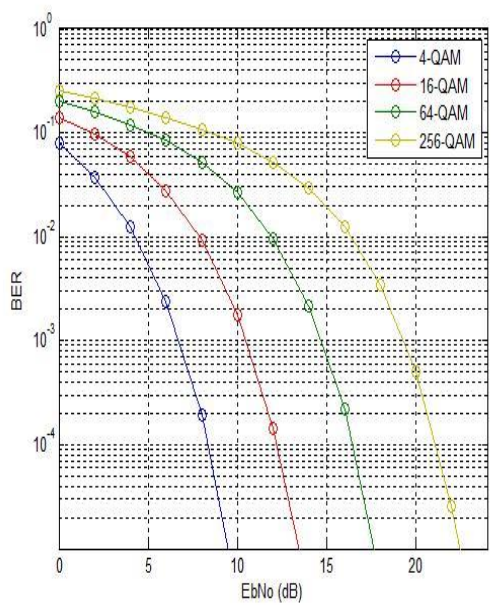

Fig. 4. The average BER as a function of SNR for the QAM modulation for $\mathrm{N}=4,16,64,256$.

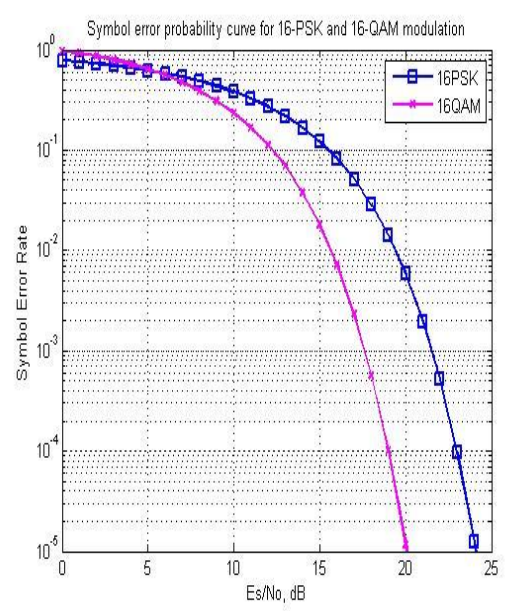

Fig: 5 SER Comparison between the QAM and QAM modulation for $\mathrm{N}=16$

Fig. 4 illustrates the error probability performance of the proposed receiver with QAM modulation for the cases where $\psi=0$, and selected values of $N$. It is assumed that the band pass filter prior to the ACD has a bandwidth of $8 \pi / T$ [10]. When $N=2$, the ACD outperforms the proposed receiver for SNR $\geq 11 \mathrm{~dB}$. Increasing the observation interval improves the performance of the proposed receiver relative to the case where $N=2$ rather rapidly. However, this increase does not have a significant impact on the performance of the ACD receiver. For example, when the average BER equals 10-6, increasing $N$ from 2 to 8 results in a 3 dB SNR gain for the proposed receiver, whereas the corresponding gain for the ACD is approximately $0.35 \mathrm{~dB}$. Fig. 3 also shows that the lower bound becomes quite tight when $N$ increases. Note that for $N=16$ and BER $=10-6$, the 
proposed receiver achieves a gain of $2.5 \mathrm{~dB}$ relative to the ACD receiver. As seen in the figure, the performance of the receiver is increasing the observation interval significantly improves the receiver performance.

Fig.5 shows the comparison of error probability performance of the proposed receiver with QAM and PSK modulation. As seen in the figure, the performance of QAM modulation is better than the PSK modulation. For example, when the SER $=10-3$, increasing SNR gain of 5dB.

\section{CONCLUSION}

A MSDD scheme was proposed for detection of DDQAM signals in the presence of random frequency variation and AWGN. It was shown that the proposed receiver is insensitive to constellation rotation due to frequency offset, and is robust to amplitude attenuation provided that the frequency offset is not large. Carrier frequency variation was shown to deteriorate the amplitude and the phase of a QAM signal transmitted over an AWGN channel. It was also shown that the proposed receiver outperforms an ACD receiver when the number of received samples contributing to the detection process becomes large. We have tested our algorithm on QAM modulation also. Those Figures are shown in above figures. We have found that the better Error probability is obtained.

\section{ACKNOWLEDGEMENT}

The authors would like to thank the anonymous reviewers of this paper for their fruitful comments and suggestions which greatly improved the quality of this paper.

\section{References}

[1] J. G. Proakis, Digital Communications, 4th edition. McGraw-Hill, 2000.

[2] D. Divsalar and M. K. Simon, "Multiple-symbol differential detectionof MPSK," IEEE Trans. Commun., vol. 38, no. 3, pp. 300-308, Mar.1990.

[3] "Maximum-likelihood differential detection of uncoded and trellis coded amplitude phase moulation over AWGN and fading channelsmetricsand performance," IEEE Trans. Commun., vol. 42, no. 1, pp.76-89, Jan. 1994.

[4] S. G. Wilson, J. Freebersyser, and C. Marshall, "Multi-symbol detectionof M-DPSK," in Proc. IEEE Global Telecommun. Conf., Nov. 1989, pp.1692-1697.

[5] A. M. Rabiei and N. C. Beaulieu, "Multiple symbol differential detectionof MPSK in the presence of frequency offset," in Proc. IEEEInt. Conf. Commun., vol. 1, May 2005, pp. 693-697.

[6] D. Divsalar and M. K. Simon, "Double differential detection," NASANew Technology Item 7170, Docket 17666, June 27; presented at IEEECommun. Theory Workshop, Apr. 1987.

[7] Y. B. Okunev, V. A. Pisarev, and V. K. Reshemkin, "The design andnoise-immunity of multiphase autocorrelation demodulators of secondorderDQAM signals," Radiotekhnika, vol. 34, no. 6, pp. 60-63, 1979;Telecomm. Radio Eng., part 2, vol. 34, no. 6, 1979, pp. 60-63.

[8] Y. B. Okunev and L. M. Fink, "Noise immunity of various receivingmethods for binary systems with second-order phase-difference modulation,"Radiotekhnika, vol. 39, no. 8, pp. 51-56, 1984; Telecommun.Radio Eng., vol. 39, no. 8, 1984, pp. 51-56.

[9] Y. B. Okunev and N. M. Sidorov, "Noise immunity of a carrierfrequency-invarient demodulator of DPSK-2 signals," Radiotekhnika,no. 6, pp. 81-83, 1986; Telecommun. Radio Eng., no. 6, 1986, pp. 81-83.

[10] M. K. Simon and D. Divsalar, "On the implementation and performanceof single and double differential detection schemes," IEEE Trans.Commun., vol. 40, no. 2, pp. 278-291, Feb. 1992.

[11] "Doppler-corrected differential detection of MPSK," IEEE Trans.Commun., vol. 37, no. 2, pp. 99-109, Feb. 1989.

[12] S. Niranjayan and N. C. Beaulieu, "On the integrated cross-noise termin correlation detectors," IEEE Trans. Commun., vol. 57, no. 11, pp.3244-3248, Nov. 2009.

[13] M. Pent, "Double differential PSK scheme in the presence of Dopplershift," in Proc. AGARD Conf. Digital Commun. Avionics, no. 239, Nov.1978, pp. 43:1-43:11.

[14] M. K. Simon and M.-S. Alouini, Digital Communication over FadingChannels, 2nd edition. Wiley, 2005.

[15] R. F. Pawula, “A new formula for MDPSK symbol error probability,"IEEE Commun. Lett., vol. 2, no. 10, pp. 271-272, Oct. 1998.

\section{AUTHORS PROFILE}

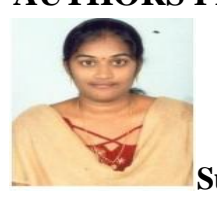

Sudharani karedla graduated from Sri Sai Aditya Institute of Science \& Technology, Surampalem in Electronics \& Communication Engineering (ECE) Stream From JNTU, Now pursuing Masters in Computers And Communication (C\&C) Stream from University college of Engineering, JNTUK, Kakinada, Andhra Pradesh, India.

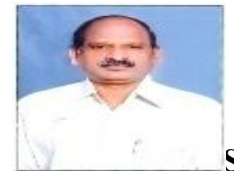

Sri E.VenkataNarayana completed B.E and M.E in first class from Andhra University, Visakhapatnam. He has been in teaching since 1985. Presently he is working as Asst. Professor in Dept. of ECE, JNTU college of Engineering Kakinada. He published more than 45 papers in national and international conferences and journals. 